\title{
AVALIAÇÃO DE HABILIDADES BÁSICAS DE ESTUDANTES NO ESPECTRO DO AUTISMO NO AMBIENTE DE APRENDIZAGEM
}

\section{EVALUATION OF BASIC SKILLS OF STUDENTS IN THE SPECTRUM OF AUTISM IN THE LEARNING ENVIRONMENT}

Francidalma Soares Sousa Carvalho Filha1, Eutima Klayre Pereira Nunes², Maria Vitória Melo de Oliveira ${ }^{3}$, Janderson Castro dos Santos ${ }^{4}$, Laylla Luanna de Mello Frasca ${ }^{5}$, Thais Vilela de Sousa ${ }^{6}$, lel Marciano de Moraes Filho ${ }^{7}$

\section{RESUMO}

Objetivo: avaliar a aquisição de habilidades básicas de estudantes no Espectro do Autismo. Método: pesquisa transversal descritiva com abordagem quantitativa. Utilizou-se como base o instrumento Avaliação da Linguagem Básica e Habilidades de Aprendizagem Revisada. Foi consentida a participação de 23 crianças no Transporto do Espectro do Autismo, em nove centros de coleta de dados. Ademais, os dados foram organizados em quadros e tabulados no software Statistical Package for the Social Sciences e, em seguida, foram consolidados por meio das técnicas de estatísticas descritivas (frequências absoluta e relativa), sendo que, posteriormente, foram discutidos com base na literatura produzida sobre o tema. Resultados: os estudantes apresentaram maior habilidade no recreio e no tempo livre, explorando brinquedos nos ambientes e retirando-os e posicionando-os de forma adequada. Nos menores níveis, apresentaram baixas habilidades na interação social, demonstrando que a maioria dos participantes não são capazes de manter contato visual, corroborando que não possuem boas experiências em grupo e que apresentam dificuldades de interação com os professores. Conclusão: os participantes da pesquisa são capazes de executar habilidades necessárias para a vida cotidiana, todavia, percebeu-se que parte dos estudantes ora não conseguia realizar a tarefa, ora obtinha baixo desempenho.

Palavras-chave: Transtorno do Espectro do Autismo. Avaliação. Habilidades.

\section{ABSTRACT}

Objective: to evaluate the acquisition of basic skills of students on the Autism Spectrum. Method: Descriptive cross-sectional research with a quantitative approach, based on the Basic Language Assessment and Revised Learning Skills instrument. 23 children were allowed to participate in the Autism Spectrum Transport, in nine data collection centers, in addition the data were organized in tables and tabulated in the Statistical Package for the Social Sciences software, then they were consolidated using the techniques of descriptive statistics (absolute and relative frequencies), were later discussed based on the literature produced on the topic. Results: the students showed greater skills in the playground and in their free time exploring toys in the environments and removing and positioning them properly and at the lowest levels they presented low skills in social interaction demonstrating that most participants are not able to maintain eye contact, corroborating that they do not have good group experiences and difficulties in interacting with teachers Conclusion: the research participants are able to perform necessary skills for daily life, however, it was noticed that part of the students were sometimes unable to perform the task, now got poor performance.

Keywords: Autism Spectrum Disorder. Evaluation. Skills.

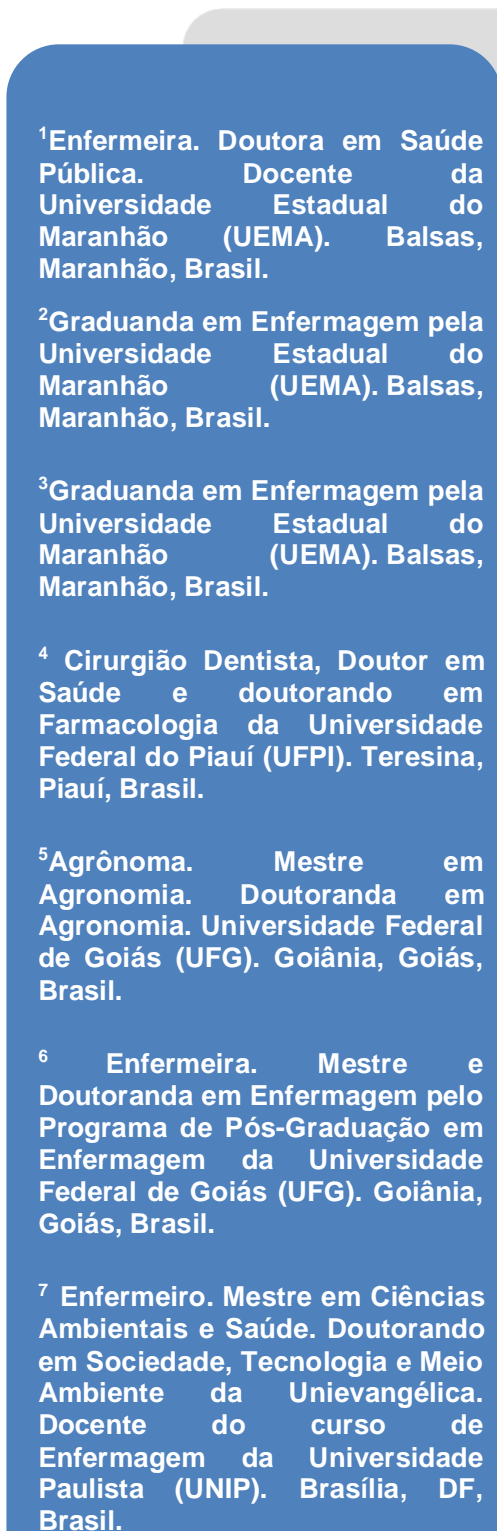

Brasil. 
DOI: 10.18606/2318-1419/amazonia.sci.health.v9n1p79-95 Revista Amazônia Science \& Health

ANO Volume/Número
CARVALHO FILHA FSS, NUNES EKP, OLIVEIRA MVM, SANTOS JC, FRASCA LLM, SOUSA TV, MORAES FILHO IM.

Avaliação de habilidades básicas de estudantes no espectro do autismo no ambiente de aprendizagem.

\section{INTRODUÇÁO}

Segundo o Manual Diagnóstico e Estatístico de Transtornos Mentais (DSM-V), o Transtorno do Espectro do Autismo (TEA) compreende déficits significativos e persistentes na interação e na comunicação social e padrões restritos e repetitivos de comportamento, de interesses ou de atividades, demonstrando uma série de manifestações de acordo com a idade e a capacidade, com as intervenções e com os apoios existentes ${ }^{1}$.

Além disso, consoante a Classificação Internacional de Funcionalidade, Incapacidade e Saúde (CIF), o autismo está incluído, no capítulo sobre funções mentais, como distúrbio que afeta a categoria das funções psicossociais globais. Essas funções desenvolvem-se ao longo da vida e são necessárias para entender e integrar funções mentais gerais, que possibilitam a criação de habilidades interpessoais necessárias ao desenvolvimento de relações sociais recíprocas. No geral, a CIF trouxe a dimensão da funcionalidade, retirando o foco do sujeito e passando a destacar também o papel do ambiente ${ }^{1}$. Dessa forma, existe uma concordância entre vários autores a respeito da tríade de dificuldades (socialização, comunicação e comportamento restrito e repetitivo) e do início precoce do TEA.

Verificado como um transtorno orgânico, consequente de uma patologia do sistema nervoso central, que, por esse motivo, compreende implicações neurobiológicas, neurofisiológicas e neuroanatômicas, o TEA também é visto como uma doença incapacitante e crônica, que provoca sérios comprometimentos no campo cognitivo e no desenvolvimento da motilidade e da linguagem, apresentando déficit ou alterações na codificação e na decodificação dos significados das palavras. Além disso, há conceitos que o definem como um impedimento neurofuncional que não permite ao seu portador 0 desenvolvimento funcional eficaz no processo de comunicação².

É fundamental que o profissional tenha bastante experiência sobre o TEA e que entenda profundamente sobre comportamentos infantis de forma geral, para que então o diagnóstico seja feito com êxito. Uma das principais dificuldades em realizar o diagnóstico precoce do autismo são as inúmeras condições clínicas adversas e comorbidades associadas, que infelizmente acabam por se confundir com o TEA ou agravar o quadro e resultar em diagnósticos equivocados, por isso é tão importante a capacitação dos 
DOI: 10.18606/2318-1419/amazonia.sci.health.v9n1p79-95 Revista Amazônia Science \& Health
CARVALHO FILHA FSS, NUNES EKP, OLIVEIRA MVM, SANTOS JC, FRASCA LLM, SOUSA TV, MORAES FILHO IM.

Avaliação de habilidades básicas de estudantes no espectro do autismo no ambiente de aprendizagem.

profissionais, para que saibam distinguir as condições clínicas características de cada transtorno e as possíveis comorbidades associadas a cada caso, por mais semelhantes que eles possam se parecer ${ }^{3}$.

Reis, Pereira e Almeida ${ }^{4}$ destacam a importância da avaliação para quem trabalha com crianças com transtornos desenvolvimentais, orientando-se sempre para a ação através de fatores como: 1) a modalidade de investigação aplicada e preocupada com a fundamentação da teoria ou com a consolidação do conhecimento já existente; 2) a comparação do desenvolvimento atual da criança com o desenvolvimento típico; e 3) a avaliação visando a fundamentação de uma tomada de decisões acerca do que se avalia, nomeadamente aprovando, rejeitando, modificando ou implementando determinadas medidas ou práticas de intervenção.

Assim, tratar-se-á de uma avaliação que não se justifica por si mesma, antes está a serviço do processo desenvolvimental e educativo da criança, informando a adequação dos seus contextos e de suas práticas educativas e buscando a melhora da convivência com outros indivíduos e até mesmo com a família, visto que os indivíduos no espectro acabam não deixando que as pessoas entrem no mundo deles, pois as suas percepções são diferentes ${ }^{5}$.

Nesse contexto, dentre os vários instrumentos de avaliação do desenvolvimento de crianças no TEA, salienta-se a Avaliação da Linguagem Básica e Habilidades de Aprendizagem Revisada (ABLLS-R), o qual é um protocolo de avaliação de indivíduos com atraso no desenvolvimento que rastreia e monitora a aquisição de 544 habilidades básicas e verbais, divididas em 25 áreas de prioridades educacionais, incluindo imitação, habilidades motoras, de falante e de ouvinte, acadêmicas, autoajuda, interação social, entre outras ${ }^{6}$.

Em consonância, um estudo realizado por Martins ${ }^{6}$ revelou que durante o treino de habilidades básicas de estudante, segundo o ABLLS-R, todos os participantes cumpriram, adequadamente, os itens propostos, como: manter contato visual por um período de 5 segundos, seguir instruções simples verbais e gestuais do experimentador, permanecer sentado na cadeira por um período de 15 minutos engajado em atividades, e aceitar o contato físico do experimentador para conduzi-los até a sala.

O ABLLS-R é composto por uma série de habilidades que estão divididas em dois documentos. Dessa forma, para esta pesquisa, foi utilizado o documento a respeito do 
protocolo que engloba as habilidades básicas de estudante, assim sendo, foram avaliadas as seguintes habilidades: de recreio e tempo livre, de interação social, de instrução grupal, de rotinas da aula e de respostas generalizadas. Os resultados da avaliação, a partir deste instrumento, permitem que os pais e os profissionais identifiquem os obstáculos que impedem a criança de adquirir novas habilidades e possibilitam 0 desenvolvimento de um currículo abrangente, altamente personalizado e baseado na linguagem ${ }^{7}$.

Logo, ressalta-se que esse documento se trata de um instrumento completo, com escalas adaptadas às necessidades de estudantes que apresentam alterações na aquisição de habilidades essenciais para o desenvolvimento de outras também essenciais, tais como a leitura e a escrita, além de prepará-los para as sociais. Portanto, avaliando estas crianças, é possível se traçar um Plano Educacional Individualizado (PEI), com vistas a se alcançar os melhores resultados possíveis. O PEI apresenta como finalidade facilitar o desenvolvimento de métodos pedagógicos que sejam semelhantes às necessidades individuais do estudante. Assim, o plano considera as dificuldades individuais dos sujeitos, fornecendo metas de acordo com suas peculiaridades ${ }^{8}$.

Deste modo, o objetivo deste trabalho foi avaliar a aquisição de habilidades básicas de estudantes no Espectro do Autismo.

\section{MATERIAIS E METODOS}

Trata-se de um estudo transversal descritivo com abordagem quantitativa. $O$ cenário desta investigação foi o município de Balsas - MA, com população estimada de 95.929 habitantes em 2021 e que apresenta uma área de unidade territorial de 13.141.757 km² e faz parte da região sul do Maranhão. A cidade é banhada pelo Rio Balsas e localiza-se a $790 \mathrm{~km}$ da capital, São Luís ${ }^{9}$.

Portanto, os locais de realização deste estudo foram as seguintes instituições: Associação de Pais e Amigos dos Excepcionais (APAE); Centro de Reabilitação em Terapia Ocupacional (CERETO) e, também, as escolas regulares de Balsas - MA, em que as crianças no TEA estão matriculadas, as quais são: Deputado Francisco Coelho; Eurico Bogea; Francisco Coelho dos Santos; Professora Virgínia Cury; Senador Alexandre Costa; Centro Educacional Aprender Pensando e Centro Educacional Sonho de Criança. 
DOI: 10.18606/2318-1419/amazonia.sci.health.v9n1p79-95 Revista Amazônia Science \& Health
CARVALHO FILHA FSS, NUNES EKP, OLIVEIRA MVM, SANTOS JC, FRASCA LLM, SOUSA TV, MORAES FILHO IM.

Avaliação de habilidades básicas de estudantes no espectro do autismo no ambiente de aprendizagem.

Logo, enfatiza-se que a APAE conta com 3 (três) crianças autistas inscritas, 0 CERETO possui 12 (doze) crianças acompanhadas e, nas escolas, existem 8 (oito) crianças que não realizam acompanhamento em nenhuma das duas instituições mencionadas. À vista disso, de posse da lista nominal das crianças no Espectro do Autismo e ciente dos dias e dos horários de atendimento agendado deles, as pessoas referidas foram procuradas e convidadas a participar desta pesquisa, sendo que, na ocasião, foram esclarecidos os objetivos e outras informações relevantes do estudo. Após a solicitação de suas participações voluntárias, somaram-se 23 participantes, que são crianças do TEA. Todos aceitaram participar da pesquisa.

Os critérios de inclusão foram: possuir diagnóstico de Transtorno do Espectro do Autismo, estar na faixa etária de cinco a quinze anos, serem acompanhadas pela APAE, CERETO e/ou estar matriculada(o) nas escolas regulares de Balsas-MA. Já os critérios de exclusão foram: não conseguir participar da avaliação, por alterações graves de comportamentos ou outras, não aceitar participar do estudo e não assinar o Termo de Assentimento Livre e Esclarecido.

A coleta de dados ocorreu no período de setembro de 2018 a março de 2019, por meio da aplicação do instrumento validado ABLLS-R, com o objetivo de avaliar as habilidades básicas presentes ou não nos estudantes avaliados. Depois, os dados coletados foram tabulados e transformados em quadros e, em seguida, compôs-se um banco de dados, os quais foram digitados no software Statistical Package for the Social Sciences - SPSS (versão 24.0 for Windows) e que, logo após, foram consolidados por meio das técnicas de estatísticas descritivas (frequências absoluta e relativa). Assim, procedeu-se a análise e a discussão dos achados com base na literatura produzida sobre o tema.

Para melhorar a compreensão dos dados, salienta-se que o ABLLS-R possui uma série de tarefas com o objetivo de avaliar, detalhadamente, o desenvolvimento das habilidades básicas de estudantes no Espectro do Autismo. Tais tarefas, que podem ser simples ou complexas, são indicadas por letras, sendo assim, as habilidades básicas avaliadas na pesquisa estão dispostas de $\mathrm{K}$ a $\mathrm{P}$.

Destarte, ao final da execução das tarefas, é registrada a pontuação, que pode alcançar o máximo de 4 pontos em determinadas tarefas, e de 2 pontos em outras. Alcança o valor de 4 o estudante que conseguir realizar completamente a tarefa sem 
ajuda; o de 3, aquele que executar a tarefa, porém com ajuda gestual ou verbal; o de 2, quem efetuar pelo menos metade da tarefa; o de 1, o participante que fizer ao menos algum item da tarefa; e o de 0, aquele que não realizar a tarefa. Quando o valor máximo é de 2 pontos, atinge este escore o participante que faz a tarefa por completo sem ajuda; quando é de 1 ponto, o estudante exerce metade da tarefa; e atinge 0 ponto, aquele que não realiza a tarefa.

Esta pesquisa foi submetida à Plataforma Brasil e, em seguida, direcionada ao Comitê de Ética em Pesquisa (CEP) do CESC-UEMA, com o Certificado de Apresentação para Apreciação Ética (CAAE), de $n^{\circ} 89324718.5 .0000 .5554$, sendo aprovada com o parecer $n^{\circ}$ 2.677.496.

\section{RESULTADOS E DISCUSSÄO}

O Quadro 1 exibe as habilidades pertinentes ao Recreio e Tempo Livre de crianças de 5 a 10 anos e de 11 a 15 anos, evidenciadas em 15 itens, identificados por meio da letra "K" - K1 a K15, as quais equivalem respectivamente a: explorar brinquedos no ambiente (K1); permitir que outros toquem nos brinquedos (K2); atividades independentes externas (K3); atividades de recreação em ambientes internos (K4); brincar com brinquedos ou usá-los apropriadamente (K5).

Foi possível observar ainda que os estudantes eram capazes de: brincar sozinhos e conversar ao mesmo tempo com os demais (K6); realizar várias interações com brinquedos relacionados a um tema (K7); brincar interagindo com outros alunos (K8); brincar de maneira interativa com uma variedade de companheiros (K9); executar jogos simbólicos (K10); brincar com os brinquedos e falar com as outras crianças (K11); realizar jogos com bola (K12); jogos liderados por outros companheiros (K13); jogos motores interativos (K14); atividades interativas de recreação (K15). 
DOI: 10.18606/2318-1419/amazonia.sci.health.v9n1p79-95 Revista Amazônia Science \& Health
CARVALHO FILHA FSS, NUNES EKP, OLIVEIRA MVM, SANTOS JC, FRASCA LLM, SOUSA TV, MORAES FILHO IM.

Avaliação de habilidades básicas de estudantes no espectro do autismo no ambiente de aprendizagem.

Quadro 1 - Dados referentes à pontuação da Habilidade de Recreio e Tempo Livre de estudantes no Espectro do Autismo, conforme a escala ABLLS-R. Balsas-MA, 2019. $(n=23)$

\begin{tabular}{|c|c|c|c|c|c|c|c|c|c|c|}
\hline \multirow{2}{*}{ Tarefas } & \multicolumn{5}{|c|}{ Estudantes de 5 a 10 anos } & \multicolumn{5}{c|}{ Estudantes de 11 a 15 anos } \\
\cline { 2 - 12 } & $\mathbf{0}$ & $\mathbf{1}$ & $\mathbf{2}$ & $\mathbf{3}$ & $\mathbf{4}$ & $\mathbf{0}$ & $\mathbf{1}$ & $\mathbf{2}$ & $\mathbf{3}$ & $\mathbf{4}$ \\
\hline $\mathrm{K} 1$ & - & 6 & 7 & - & - & - & - & 10 & - & - \\
\hline $\mathrm{K} 2$ & - & 6 & 7 & - & - & - & - & 10 & - & - \\
\hline $\mathrm{K} 3$ & 2 & 2 & 3 & 2 & 4 & - & 1 & - & 1 & 8 \\
\hline $\mathrm{K} 4$ & 2 & 2 & 1 & 3 & 5 & 1 & - & - & 1 & 8 \\
\hline $\mathrm{K} 5$ & 3 & 4 & 6 & - & - & 1 & 1 & 8 & - & - \\
\hline $\mathrm{K} 6$ & 2 & 3 & 4 & 3 & 1 & - & 1 & 1 & - & 8 \\
\hline $\mathrm{K} 7$ & 2 & 4 & 1 & 6 & - & 1 & - & 1 & - & 8 \\
\hline $\mathrm{K} 8$ & 2 & 4 & 7 & - & - & 1 & - & 2 & 1 & 6 \\
\hline $\mathrm{K} 9$ & 2 & 3 & 6 & 2 & - & 1 & 2 & - & - & 7 \\
\hline $\mathrm{K} 10$ & 5 & 5 & 1 & 2 & - & 2 & - & - & 1 & 7 \\
\hline $\mathrm{K} 11$ & 3 & 4 & 4 & 2 & - & 1 & - & 2 & - & 7 \\
\hline $\mathrm{K} 12$ & 3 & 1 & 4 & 5 & - & - & 1 & 1 & 1 & 7 \\
\hline $\mathrm{K} 13$ & 3 & 5 & 1 & 4 & - & 1 & - & 1 & 1 & 7 \\
\hline $\mathrm{K} 14$ & 2 & 4 & 4 & 3 & - & - & 1 & - & 1 & 8 \\
\hline $\mathrm{K} 15$ & 4 & 1 & 3 & 5 & - & 1 & - & 1 & - & 8 \\
\hline
\end{tabular}

Fonte: Pesquisa direta, 2019.

No tocante à Tarefa K1 (explorar brinquedos no ambiente), verificou-se que 6 $(26,1 \%)$ atingiram o escore 1 , e $17(73,9 \%)$, o escore 2 . Assim, pode-se dizer que a maior parte dos estudantes consegue explorar brinquedos no ambiente, de forma independente. $\mathrm{Na}$ Tarefa K5 (brincar com brinquedos ou usá-los apropriadamente), constatou-se que 4 $(17,4 \%)$ estudantes obtiveram o escore $0 ; 5(21,7 \%)$, o escore 1 ; e 14 (60,9\%), o escore 2 , sendo que a prevalência foi dos participantes que executaram a tarefa satisfatoriamente.

De acordo com a Tarefa K15 (atividades interativas de recreação), observou-se que $5(21,7 \%)$ participantes marcaram o escore $0 ; 1(4,3 \%)$, o escore $1 ; 4(17,4 \%)$, o escore 2; 5 (21,7\%), o escore 3; e 8 (34,9\%), o escore 4 , significando que os participantes estão aptos a realizar atividades interativas de recreação.

Contudo, averiguou-se que os participantes, em sua maioria, demonstram pontuações adequadas, exprimindo ainda que são detentores da habilidade de recreio e tempo livre, assim, essa informação é configurada como positiva. Tendo isso em vista, 
DOI: 10.18606/2318-1419/amazonia.sci.health.v9n1p79-95 Revista Amazônia Science \& Health
CARVALHO FILHA FSS, NUNES EKP, OLIVEIRA MVM, SANTOS JC, FRASCA LLM, SOUSA TV, MORAES FILHO IM.

Avaliação de habilidades básicas de estudantes no espectro do autismo no ambiente de aprendizagem.

salienta-se que as brincadeiras favorecem a interação social das crianças, bem como auxiliam no desenvolvimento da interação motora grossa e da cognição.

Ferreira ${ }^{10}$ comenta que frequentemente é referido pelos pais de crianças com TEA que estas apresentam uma forma de brincar diferente da apresentada, por exemplo, pelos seus irmãos na mesma idade. Normalmente, a criança com TEA centra-se apenas em partes do brinquedo, isto é, se for, por exemplo, um brinquedo que haja um fio de puxar para fazer andar, a criança fixa-se no movimento de puxá-lo, mas não no que isso vai provocar. Por outro lado, o interesse por um brinquedo tende a ser reduzido, ou seja, a criança o observa e pouco depois acaba por largá-lo.

Desta maneira, segundo Cruz ${ }^{11}$, é no "brincar" que as crianças começam a interagir com os objetos, com as pessoas e com o mundo ao seu redor. O autor afirma ainda que, embora algumas crianças autistas pareçam brincar, elas se preocupam mais em alinhar e manusear brinquedos do que em usá-los para a sua finalidade simbólica.

O Quadro 2 expõe as habilidades relativas à Interação Social de crianças de 5 a 10 anos e de 11 a 15 anos, citadas em 30 itens, nomeados pela letra "L" - L1 a L20, representados na devida ordem de: manter comportamento adequado quando estiver perto de outras crianças e de seus irmãos (L1); aceitar objetos oferecidos (L2); responder apropriadamente ao contato físico positivo de companheiros e de irmãos (L3); mostrar interesse pelo comportamento dos outros (L4); olhar para outros para iniciar uma interação social (L5); aproximar-se fisicamente e atrair outros (L6).

Ainda neste quadro, investigou-se se o participante estaria apto a: olhar de expectativa para outros antes de obter um reforço (L7); seguir instruções simples (L8); imitar os companheiros (L9); responder a cumprimentos (L10); sugerir fisicamente para que outros façam atividades (L11); responder a aproximações e tentativas de interação com seus companheiros (L12); compartilhar - dar objetos a outros (L13); procurar uma pessoa ausente (L14); buscar atenção de outros (L15); nomear objetos para outras pessoas (L16); olhar nos olhos (L17); pedir para que os colegas dividam os objetos com ele (L18); compartilhar objetos de maneira espontânea (L19); participar com os colegas em uma atividade (L20). 
DOI: 10.18606/2318-1419/amazonia.sci.health.v9n1p79-95 Revista Amazônia Science \& Health
CARVALHO FILHA FSS, NUNES EKP, OLIVEIRA MVM, SANTOS JC, FRASCA LLM, SOUSA TV, MORAES FILHO IM.

Avaliação de habilidades básicas de estudantes no espectro do autismo no ambiente de aprendizagem.

Quadro 2 - Dados referentes à pontuação da Habilidade de Interação Social de estudantes no Espectro do Autismo, conforme a escala ABLLS-R. Balsas-MA, 2019. $(n=23)$

\begin{tabular}{|c|c|c|c|c|c|c|c|c|c|c|}
\hline \multirow[t]{2}{*}{ Tarefas } & \multicolumn{5}{|c|}{$\begin{array}{l}\text { Estudantes de } 5 \text { a } 10 \text { anos } \\
\qquad N=13\end{array}$} & \multicolumn{5}{|c|}{$\begin{array}{l}\text { Estudantes de } 11 \text { a } 15 \text { anos } \\
\qquad N=10\end{array}$} \\
\hline & 0 & 1 & 2 & 3 & 4 & 0 & 1 & 2 & 3 & 4 \\
\hline L1 & 3 & 1 & 4 & 4 & 1 & 1 & - & - & 1 & 8 \\
\hline L2 & - & 8 & 5 & - & - & - & 2 & 8 & - & - \\
\hline L3 & 2 & 10 & 1 & - & - & 1 & 2 & 7 & - & - \\
\hline L4 & 4 & 5 & 4 & - & - & 1 & 7 & 2 & - & - \\
\hline L5 & 2 & 10 & 1 & - & - & 1 & 2 & 7 & - & - \\
\hline L6 & 3 & 10 & - & - & - & 1 & 2 & 7 & - & - \\
\hline L7 & 3 & 5 & 5 & - & - & 1 & - & 9 & - & - \\
\hline L8 & 3 & 7 & 3 & - & - & 1 & 1 & 8 & - & - \\
\hline L9 & 6 & 3 & 4 & - & - & - & 2 & 8 & - & - \\
\hline L10 & 5 & 2 & 6 & - & - & 2 & - & 1 & 2 & 5 \\
\hline L11 & 2 & 11 & - & - & - & - & 3 & 7 & - & - \\
\hline L12 & 4 & 7 & 2 & - & - & 1 & 2 & 7 & - & - \\
\hline L13 & 3 & 9 & 1 & - & - & - & 3 & 7 & - & - \\
\hline L14 & 4 & 5 & 4 & - & - & 1 & 2 & 7 & - & - \\
\hline L15 & 4 & 2 & 4 & - & 3 & 1 & - & 1 & 2 & 6 \\
\hline L16 & 5 & 5 & 3 & - & - & 2 & 7 & 1 & - & - \\
\hline L17 & 9 & - & 2 & 2 & - & 1 & 1 & 8 & - & - \\
\hline L18 & 6 & 4 & 3 & - & - & 1 & - & 2 & 2 & 5 \\
\hline L19 & 4 & 9 & - & - & - & 2 & 6 & 2 & - & - \\
\hline L20 & 4 & 9 & - & - & - & 2 & 7 & 1 & - & - \\
\hline
\end{tabular}

Fonte: Pesquisa direta, 2019.

Conforme se percebe no quadro acima, na Tarefa L6 (aproximar-se fisicamente e atrair outros), 4 (17,4\%) participantes obtiveram escore $0 ; 12$ (52,2\%), escore 1 ; e 7 $(30,4 \%)$ escore 2 , mostrando que prevaleceram os estudantes que só conseguiram realizar a tarefa mediante ajuda. A respeito da Tarefa L11 (sugerir fisicamente para que outros façam atividades), 2 (8,7\%) participantes tiveram o escore $0 ; 14$ (60,9\%), o escore 1 ; e $7(30,4 \%)$, o escore 2 , evidenciando que a quantidade majoritária destes estudantes teve um baixo desempenho.

De acordo com a Tarefa L17 (olhar nos olhos), verificou-se que 10 (43,5\%) apresentaram escore $0 ; 1$ (4,3\%), escore $1 ; 10$ (43,5\%), escore 2; e 2 (8,7\%), escore 3. Estes dados demonstram que a maioria dos participantes não são capazes de manter contato visual. Mediante os dados expostos, comprovou-se que a maior parte dos 
DOI: 10.18606/2318-1419/amazonia.sci.health.v9n1p79-95 Revista Amazônia Science \& Health

ANO Volume/Número
CARVALHO FILHA FSS, NUNES EKP, OLIVEIRA MVM, SANTOS JC, FRASCA LLM, SOUSA TV, MORAES FILHO IM.

Avaliação de habilidades básicas de estudantes no espectro do autismo no ambiente de aprendizagem.

estudantes apresenta prejuízos na interação social, tal declaração é feita por esta ser uma das principais características do transtorno.

Dessa forma, pode se considerar que a maior dificuldade da criança autista se encontra em sua capacidade de vivenciar a reciprocidade contida nas interações, quando não apresenta a capacidade de agir e de aguardar a reação de seu companheiro de interação. Tal dificuldade prejudica severamente o estabelecimento das interações sociais e acarreta também o prejuízo no aparecimento de muitos comportamentos, como o contato ocular, o sorriso responsivo e as vocalizações utilizadas para a comunicação ${ }^{12}$.

Para interagir efetivamente com as pessoas, são necessárias habilidades sociais que englobam a capacidade de dividir espaços com outros de maneira adequada, de adaptar-se a diferentes contextos e de interpretar pensamentos e desejos dos outros. Dessa maneira, percebe-se o quanto essa habilidade é essencial para a interação e, também, para o comportamento adequado em diferentes contextos, como os da sala de aula e do ambiente de trabalho, entre outros ${ }^{11}$.

O Quadro 3 denota as habilidades relativas à Instrução Grupal de crianças de 5 a 10 anos e de 11 a 15 anos, dispostos em 12 itens, identificados pela letra "M" - M1 a M12, caracterizados por: sentar-se adequadamente em um grupo pequeno (M1); sentarse adequadamente em um grupo grande (M2); responder ao professor em grupo (M3); responder a outros estudantes em grupo (M4); seguir instruções em grupo (M5); seguir instruções em grupo com descrições (M6); levantar a mão para ter a atenção do professor, antes de realizar alguma atividade (M7); levantar a mão para responder a alguma pergunta (M8); levantar a mão para dar nome a algum objeto (M9); levantar a mão e responder a uma pergunta (M10); revezar/trocar (turnos) durante um treinamento (M11); aprender novas habilidades a partir de um ensinamento de grupo (M12).

Quadro 3 - Dados referentes à pontuação da Habilidade de Instrução Grupal de estudantes no Espectro do Autismo, conforme a escala ABLLS-R. Balsas-MA, 2019. $(n=23)$

\begin{tabular}{|c|c|c|c|c|c|c|c|c|c|c|}
\hline \multirow[t]{2}{*}{ Tarefas } & \multicolumn{5}{|c|}{$\begin{array}{l}\text { Estudantes de } 5 \text { a } 10 \text { anos } \\
\qquad N=13\end{array}$} & \multicolumn{5}{|c|}{$\begin{array}{l}\text { Estudantes de } 11 \text { a } 15 \text { anos } \\
\qquad N=10\end{array}$} \\
\hline & 0 & 1 & 2 & 3 & 4 & 0 & 1 & 2 & 3 & 4 \\
\hline M1 & 3 & 1 & 5 & 3 & 1 & 1 & - & - & 3 & 6 \\
\hline M2 & 8 & 1 & 3 & 1 & - & 1 & 1 & - & 2 & 6 \\
\hline M3 & 7 & 6 & - & - & - & 1 & 1 & - & 5 & 3 \\
\hline
\end{tabular}


DOI: 10.18606/2318-1419/amazonia.sci.health.v9n1p79-95 Revista Amazônia Science \& Health

ANO Volume/Número
CARVALHO FILHA FSS, NUNES EKP, OLIVEIRA MVM, SANTOS JC, FRASCA LLM, SOUSA TV, MORAES FILHO IM.

Avaliação de habilidades básicas de estudantes no espectro do autismo no ambiente de aprendizagem.

\begin{tabular}{|l|l|l|l|l|l|l|l|l|l|l|}
\hline M4 & 7 & 6 & - & - & - & 1 & 1 & 4 & 3 & 1 \\
\hline M5 & 4 & 8 & 1 & - & - & 1 & 1 & 7 & - & 1 \\
\hline M6 & 6 & 7 & - & - & - & 1 & 1 & 6 & 1 & 1 \\
\hline M7 & 7 & 6 & - & - & - & 2 & 7 & 1 & - & - \\
\hline M8 & 7 & 6 & - & - & - & 2 & 7 & 1 & - & - \\
\hline M9 & 7 & 6 & - & - & - & 2 & 7 & 1 & - & - \\
\hline M10 & 7 & 6 & - & - & - & 2 & 7 & 1 & - & - \\
\hline M11 & 7 & 6 & - & - & - & 2 & 7 & 1 & - & - \\
\hline M12 & 6 & 6 & 1 & - & - & 2 & 7 & 1 & - & - \\
\hline
\end{tabular}

Fonte: Pesquisa direta, 2019.

Relativo à Tarefa M5 (seguir instruções em grupo), 5 (21,7\%) dos participantes obtiveram o escore 0; 9 (39,2\%), o escore 1; 8 (34,8\%), o escore 2; e 1 (4,3\%), o escore 4. Verificou-se, portanto, que a maioria dos estudantes conseguiu seguir instruções em grupo somente através de ajuda. Em relação à Tarefa M12 (aprender novas habilidades a partir de um ensinamento de grupo), notou-se que $8(34,8 \%)$ participantes tiveram escore $0 ; 13$ (56,5\%), escore 1; e 2 (8,7\%), escore 2, configurando um predomínio dos que não conseguiram desenvolver a atividade.

As informações acima revelam que os estudantes avaliados tiveram pontuações baixas, demonstrando que não possuem boas experiências em grupo, o que pode ser relacionado aos baixos escores alcançados pelos participantes na habilidade de interação social, pois, para uma habilidade se manter adequada, requer a outra. Assim, faz-se necessário incluir estes alunos em atividades que estimulem o contato com o outro, a fim de desenvolver esta habilidade.

Nesse ínterim, ressalta-se que crianças no TEA apresentam déficits consideráveis em sua capacidade de usar os significados firmados das palavras de seu grupo social, como o uso da fala espontânea e os prejuízos na capacidade de compreender e de adquirir regras e convenções sociais. Além disso, tais fatores culminam com os prejuízos na capacidade de autoconsciência, na medida em que a criança autista não se baseia na resposta afetiva do outro para refletir acerca de seus próprios comportamentos e ações ${ }^{13}$.

Assim, a maior porção das crianças no TEA possui alterações nos relacionamentos, podendo apresentar agressividade, isolamento, resistência em participar de atividades grupais e problemas com o uso funcional do brincar funcionalmente. Além disso, pode ter muita dificuldade em se imaginar no lugar do outro, não compreendendo o que o outro espera dela ${ }^{13}$. 
DOI: 10.18606/2318-1419/amazonia.sci.health.v9n1p79-95 Revista Amazônia Science \& Health
CARVALHO FILHA FSS, NUNES EKP, OLIVEIRA MVM, SANTOS JC, FRASCA LLM, SOUSA TV, MORAES FILHO IM.

Avaliação de habilidades básicas de estudantes no espectro do autismo no ambiente de aprendizagem.

O Quadro 4 identifica as habilidades relativas às Rotinas de Aula de crianças de 5 a 10 anos e de 11 a 15 anos, em 10 itens, indicados através da letra "N" - N1 a N10, que se dividem em: seguir rotinas diárias (N1); trabalhar de maneira independente em rotinas não acadêmicas (N2); sentar e esperar, de forma adequada, a transição de uma atividade para outra (N3); mudar de atividade ou se mover em outro contexto (N4); esperar a sua vez durante as atividades (N5); estar em uma fila (N6); trabalhar de maneira independente em rotinas acadêmicas (N7); retirar e posicionar os materiais de forma adequada (N8); completar uma tarefa, entregar o trabalho para o professor e guardar os materiais (N9); preparar-se e esperar, adequadamente, as transições (N10).

Quadro 4 - Dados referentes à pontuação das Habilidades de Rotinas de Aula de estudantes no Espectro do Autismo, conforme a escala ABLLS-R. Balsas-MA, 2019. $(n=23)$

\begin{tabular}{|c|c|c|c|c|c|c|c|c|c|c|}
\hline \multirow{2}{*}{ Tarefas } & \multicolumn{4}{|c|}{ Estudantes de 5 a 10 anos } & \multicolumn{5}{c|}{ Estudantes de 11 a 15 anos } \\
\cline { 2 - 12 } & $\mathbf{0}$ & $\mathbf{1}$ & $\mathbf{2}$ & $\mathbf{3}$ & $\mathbf{4}$ & $\mathbf{0}$ & $\mathbf{1}$ & $\mathbf{2}$ & $\mathbf{3}$ & $\mathbf{4}$ \\
\hline N1 & 6 & 6 & 1 & - & - & 1 & - & 9 & - & - \\
\hline N2 & 4 & 1 & 4 & 3 & 1 & 1 & - & 2 & 2 & 5 \\
\hline N3 & 4 & 4 & 5 & - & - & 1 & - & 9 & - & - \\
\hline N4 & 5 & 8 & - & - & - & 1 & 1 & 8 & - & - \\
\hline N5 & 3 & 10 & - & - & - & 1 & 1 & 8 & - & - \\
\hline N6 & 5 & 8 & - & - & - & 1 & 1 & 8 & - & - \\
\hline N7 & 4 & 4 & 1 & 3 & 1 & 1 & - & 2 & 2 & 6 \\
\hline N8 & 2 & 8 & 3 & - & - & 1 & 1 & 8 & - & - \\
\hline N9 & 4 & 9 & - & - & - & 1 & 1 & 8 & - & - \\
\hline N10 & 3 & 10 & - & - & - & 1 & 1 & 8 & - & - \\
\hline
\end{tabular}

Fonte: Pesquisa direta, 2019.

De acordo com a Tarefa N5 (esperar a sua vez durante as atividades), foi possível analisar que $4(17,4 \%)$ participantes possuíram o escore $0 ; 11(47,9 \%)$, o escore 1 ; e 8 $(34,7 \%)$, o escore 2 . Desse modo, considera-se que a maioria realizou a atividade através de ajuda. Concernente à Tarefa N8 (retirar e posicionar os materiais de forma adequada), percebe-se que $3(13,0 \%)$ tiveram escore $0 ; 9$ (39,2\%) alcançaram escore 1, e $11(47,8 \%)$ atingiram escore 2. Diante disso, alega-se que a maior parte retirou e posicionou os materiais de forma adequada, no entanto, o percentual dos que não realizaram a atividade corretamente também foi expressivo. 
DOI: 10.18606/2318-1419/amazonia.sci.health.v9n1p79-95 Revista Amazônia Science \& Health
CARVALHO FILHA FSS, NUNES EKP, OLIVEIRA MVM, SANTOS JC, FRASCA LLM, SOUSA TV, MORAES FILHO IM.

Avaliação de habilidades básicas de estudantes no espectro do autismo no ambiente de aprendizagem.

Desse modo, a tríade sintomatológica que caracteriza o perfil da pessoa com autismo não pode ser ignorada no contexto da escola. As especificidades do transtorno podem interferir no aprendizado e na inclusão social desses indivíduos em ambientes educacionais. A proximidade física com os colegas, a dificuldade em aprender regras sociais, a falta de compreensão de instruções verbais e a incapacidade em utilizar a linguagem falada podem representar desafios para essa população ${ }^{14,15}$.

Ademais, muitos dos prejuízos cognitivos associados a problemas de comportamento e de comunicação interferem consideravelmente na adaptação de uma criança à escola e a contextos sociais e familiares em geral. Elas apresentam dificuldades para resolver problemas da vida diária, para comunicar-se de maneira eficaz, para manter relacionamentos sociais e para lidar com imprevistos e dificuldades diárias. Em nível variado, as crianças podem ter dificuldades em: segmentar tarefas; organizar o tempo de trabalho durante longos períodos até uma meta final; compreender as informações /instruções relacionadas a uma tarefa; planejar e organizar e, quando necessário, solicitar ajuda do professor ${ }^{14}$.

O Quadro 5 evidencia as habilidades relativas às Respostas Generalizadas de crianças de 5 a 10 anos e de 11 a 15 anos, em 6 itens, classificados pela letra "P" - P1 a $\mathrm{P} 6$, estabelecidos em: generalizar vários estímulos (P1); generalizar vários professores (P2); generalizar vários ambientes (P3); mostrar habilidades em grupo (P4); apresentar formas generalizadas de responder (P5); mostrar generalização de habilidades de linguagem (P6).

Quadro 5 - Dados referentes à pontuação das Habilidades de Respostas Generalizadas de estudantes no Espectro do Autismo, conforme a escala ABLLS-R. Balsas-MA, 2019. $(n=23)$

\begin{tabular}{|c|c|c|c|c|c|c|c|c|c|c|}
\hline \multirow[t]{2}{*}{ Tarefas } & \multicolumn{5}{|c|}{$\begin{array}{l}\text { Estudantes de } 5 \text { a } 10 \text { anos } \\
N=13\end{array}$} & \multicolumn{5}{|c|}{$\begin{array}{l}\text { Estudantes de } 11 \text { a } 15 \text { anos } \\
N=10\end{array}$} \\
\hline & 0 & 1 & 2 & 3 & 4 & 0 & 1 & 2 & 3 & 4 \\
\hline $\mathrm{P} 1$ & 4 & 7 & 2 & - & - & 1 & 2 & 7 & - & - \\
\hline P2 & 4 & 8 & 1 & - & - & 1 & 2 & 7 & - & - \\
\hline P3 & 4 & 6 & 3 & - & - & 1 & 1 & 8 & - & - \\
\hline $\mathrm{P} 4$ & 5 & 7 & 1 & - & \begin{tabular}{|l}
- \\
\end{tabular} & 1 & 1 & 8 & \begin{tabular}{|l}
- \\
\end{tabular} & - \\
\hline P5 & 5 & 8 & - & - & - & 1 & 1 & 8 & - & - \\
\hline $\mathrm{P} 6$ & 6 & 6 & 1 & - & \begin{tabular}{|l}
- \\
\end{tabular} & \begin{tabular}{|l}
1 \\
\end{tabular} & 1 & 8 & \begin{tabular}{|l}
- \\
\end{tabular} & - \\
\hline
\end{tabular}

Fonte: Pesquisa direta, 2019. 
DOI: 10.18606/2318-1419/amazonia.sci.health.v9n1p79-95 Revista Amazônia Science \& Health
CARVALHO FILHA FSS, NUNES EKP, OLIVEIRA MVM, SANTOS JC, FRASCA LLM, SOUSA TV, MORAES FILHO IM.

Avaliação de habilidades básicas de estudantes no espectro do autismo no ambiente de aprendizagem.

Com base nas informações acima, identificou-se que, na Tarefa P2 (generalizar vários professores), 5 (21,7\%) estudantes obtiveram escore $0 ; 10$ (43,5\%), escore 1 ; e 8 $(34,8 \%)$, escore 2 , demonstrando uma prevalência de desempenho insatisfatório. Acerca da Tarefa P5 (formas generalizadas de responder), notou-se que 6 (26,1\%) possuíram o escore 0; 9 (39,1\%), o escore 1; e 8 (34,8\%), o escore 2, revelando que a maior parte dos participantes não realizou a atividade completamente. Dessa maneira, percebe-se que esta habilidade está prejudicada nestes participantes, indicando que eles possuem dificuldade de interagir com os professores e em grupo, além dos prejuízos que podem comprometer a aprendizagem.

Referente a isso, a generalização, portanto, não é algo que deva ser esperado, mas também deve ser incluída como um dos objetivos de ensino. Adaptar os métodos de treino em habilidades sociais para as crianças com TEA é complexo, pois o prejuízo social dessas crianças é qualitativamente diferente dos outros transtornos psiquiátricos da infância. Devido à dificuldade que muitos indivíduos do espectro apresentam em relação à generalização, alguns pesquisadores apontam que é importante que os treinos sejam realizados no ambiente natural da criança, como em casa e na escola ${ }^{15-17}$.

As limitações deste estudo assentam-se no fato de existirem poucas literaturas discutindo a respeito da avaliação das habilidades no cotidiano escolar de estudantes com TEA e da pesquisa ter sido realizada em um único município, com um número pequeno de participantes. Ademais, não deixa de ser relevante, devido à inexistência de outros estudos a um público tão carente de práticas inovadoras para a consolidação de seus aspectos humanos desenvolvimentais.

\section{CONSIDERAÇOES FINAIS}

Após a avaliação das 23 crianças, através do instrumento ABLLS-R, constatou-se que os estudantes apresentaram maior habilidades no recreio e no tempo livre, explorando brinquedos nos ambientes, retirando-os e posicionando-os de forma adequada e, nos menores níveis, apresentaram baixas habilidades na Interação Social, demonstrando que a maioria dos participantes não é capaz de manter contato visual, corroborando que não possuem boas experiências em grupo e apresentam dificuldades de interação com os professores. 
DOI: 10.18606/2318-1419/amazonia.sci.health.v9n1p79-95 Revista Amazônia Science \& Health
CARVALHO FILHA FSS, NUNES EKP, OLIVEIRA MVM, SANTOS JC, FRASCA LLM, SOUSA TV, MORAES FILHO IM.

Avaliação de habilidades básicas de estudantes no espectro do autismo no ambiente de aprendizagem.

Teoricamente, é notório que os participantes da pesquisa são capazes de executar habilidades necessárias para a vida cotidiana, todavia, percebeu-se que parte dos estudantes ora não conseguia realizar a tarefa, ora obtinha baixo desempenho. Aos que não tiveram bons resultados, ressalta-se que estes devem se tornar foco de intervenções que venham a inibir e/ou minimizar as características marcantes do transtorno.

Portanto, conclui-se que a pesquisa foi capaz de identificar em quais habilidades básicas as crianças apresentam dificuldades ou facilidades, assim sendo, sugere-se que avaliações como esta sejam aplicadas em estudantes no Espectro do Autismo, com o fito de identificar em quais áreas eles apresentam dificuldades e, assim, elaborar um PEI, uma vez que, este é uma ferramenta metodológica que auxilia no processo de aprendizagem considerando as particularidades dos estudantes.

\section{REFERÉNCIAS}

1.APA - American Psychiatric Associaton. Manual Diagnóstico e Estatístico de Transtornos Mentais DSM-5. Porto Alegre: Artmed, 2014.

2.Silva, MVT. Trajetórias escolares de alunos com transtorno do espectro autista e expectativas educacionais das famílias [dissertação]. Campinas: Universidade Estadual de Campinas. 2014. 119 p.

3.Pessim L, Fonseca B. Transtornos do espectro autista: importância e dificuldade do diagnóstico precoce. Revista FAEF. 2015; 3(14): 7-28. Disponível em: http://faef.revista.inf.br/imagens_arquivos/arquivos_destaque/pnnWsCHLoL9zOLE2015-33-14-7-28.pdf

4.Reis HIS, Pereira APS, Almeida LS. Construção e validação de um instrumento de avaliação do perfil desenvolvimental de crianças com perturbação do espectro do autismo. Rev. bras. educ. espec.. 2013;19(2):183-194. DOI : http://dx.doi.org/10.1590/S1413-65382013000200004.

5.Souza RFA, Souza JCP. Os desafios vivenciados por famílias de crianças diagnosticadas com Transtorno de Espectro Autista. Perspectivas em Diálogo $.2021 ; 8(16): \quad 164-182 \quad . \quad$ Disponíve

https://periodicos.ufms.br/index.php/persdia/article/view/10668

6.Martins, GT. Avaliação de dois protocolos de ensino de relações de identidade generalizada em indivíduos com atraso severo no desenvolvimento [dissertação]. Brasília: Universidade de Brasília. 2010. 77 p. 
DOI: 10.18606/2318-1419/amazonia.sci.health.v9n1p79-95 Revista Amazônia Science \& Health

ANO Volume/Número
CARVALHO FILHA FSS, NUNES EKP, OLIVEIRA MVM, SANTOS JC, FRASCA LLM, SOUSA TV, MORAES FILHO IM.

Avaliação de habilidades básicas de estudantes no espectro do autismo no ambiente de aprendizagem.

7.Partignton, JW. Guía de ABLLS-R: Avaliación de Habilidades de Lenguajie Y Aprendizaje Básicos (ABLLS-R). Version 2.0. Behavior Analysts: Walnut Creek, Califórnia,2013.

8.Mascaro CAAC. O Plano Educacional Individualizado e o estudante com deficiência intelectual: estratégia para inclusão. REA. 2018;18(205):12-22. Disponível em: http://www.periodicos.uem.br/ojs/index.php/EspacoAcademico/article/view/43318

9.Instituto Brasileiro de Geografia e Estatística. Cidades. Disponível em: <http:// www.ibge.gov.br/cidades>. Acesso em: 15 de fev. de 2021.

10.Ferreira MMJM. A integração dos pais na intervenção de crianças com autismo [monografia]. Porto: Universidade Fernando Pessoa. 2011. 66 p.

11.Cruz C, Pereira C, Ferreira C, Santos H, Ribeiro M. Criança autista: pais e professores-uma parceria de sucesso no desenvolvimento de competências. Millenium. 2010; 1(39):89-107. Disponível: https://revistas.rcaap.pt/millenium/article/view/8234

12.Correia OF, Lampreia C. A conexão afetiva nas intervenções desenvolvimentistas para crianças autistas. Psicol. cienc. prof.. 2012; 32(4):926-941. Doi : http://dx.doi.org/10.1590/S1414-98932012000400012.

13.Khoury LP, Teixeira MCTV, Carreiro LRR, Schwartzman JS, Ribeiro AF, Cantiere CN. Manejo comportamental de crianças com Transtornos do Espectro do Autismo em condição de inclusão escolar: guia de orientação a professores [livro eletrônico]. -- São Paulo: Memnon, 2014.

14.Leitão, PB. Transtorno do Espectro do Autismo na perspectiva do ensino estruturado. Pedagogia em Ação. 2016; 8(2):1-15. Disponível em: http://periodicos.pucminas.br/index.php/pedagogiacao/article/view/12865

15.Gomes RC, Nunes DRP. Interações comunicativas entre uma professora e um aluno com autismo na escola comum: uma proposta de intervenção. Educ. Pesqui.. 2014; 40(1):143-161. Doi : http://dx.doi.org/10.1590/S1517-97022014000100010.

16.Carvalho Filha FSS, Castro RP, Vilanova JM, Silva MVRS, Moraes Filho IM, Sousa TVS. Aplicação da teoria de Callista Roy a pais/Cuidadores de crianças autistas: uma proposta intervencionista. Rev Enferm Atual Derme.94(32):e-20081. Doi: https://doi.org/10.31011/reaid-2020-v.94-n.32-art.728

17.Jesus JC, Oliveira T P, Rezende Junio Vi. Generalização de mandos aprendidos pelo PECS (Picture Exchange Communication System) em crianças com transtorno do 
DOI: 10.18606/2318-1419/amazonia.sci.health.v9n1p79-95 Revista Amazônia Science \& Health ANO Volume/Número
CARVALHO FILHA FSS, NUNES EKP, OLIVEIRA MVM, SANTOS JC, FRASCA LLM, SOUSA TV, MORAES FILHO IM.

Avaliação de habilidades básicas de estudantes no espectro do autismo no ambiente de aprendizagem.

espectro autista. Temas psicol. 2017; 25(2): 531-543. Doi: http://dx.doi.org/10.9788/TP2017.2-07. 